\title{
Perturbative simulations of crack front waves
}

\author{
John W. Morrissey ${ }^{\mathrm{a}}$, James R. Rice ${ }^{\mathrm{b}, *}$

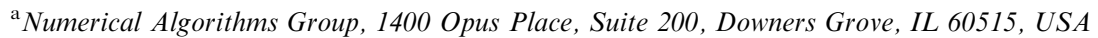 \\ ${ }^{\mathrm{b}}$ Division of Engineering and Applied Sciences and Department of Earth and Planetary Sciences, \\ Harvard University, 29 Oxford Street, Cambridge, MA 02138, USA
}

Received 1 February 1999; received in revised form 29 July 1999

\begin{abstract}
Willis and Movchan [Willis, J.R., Movchan, A.B., 1995. Dynamic weight functions for a moving crack I. Mode I loading. J. Mech. Phys. Solids 43, 319.] devised weight functions for a dynamic mode I fracture, within the singular crack model, using a first order perturbation of in-plane crack motion from the 2D results. Ramanathan and Fisher [Ramanathan, S., Fisher, D.S., 1997. Dynamics and instabilities of planar tensile cracks in heterogeneous media. Phys. Rev. Lettr. 79, 877.] reformulated the Willis-Movchan's result in terms of crack growth at constant fracture energy, thereby confirming the existence of a crack front wave. Such a wave, as a propagating mode local to the moving crack front, was seen in the non-perturbative numerical simulations based on a cohesive zone fracture model, equivalent to growth at constant fracture energy. In this paper, the result of Ramanathan and Fisher, given in the wavenumber-frequency domain, is recast in the wavenumber-time domain to analyze fracture propagation within first-order perturbations for the singular crack model. This allows application of a spectral numerical methodology and is shown to be consistent with the known 2D results. Through analysis of a single spatial mode of crack shape, the propagating crack front wave and its resonance are demonstrated. Crack propagation through a randomly heterogeneous zone, and growth of disorder with propagation distance, are also examined. (C) 2000 Elsevier Science Ltd. All rights reserved.
\end{abstract}

Keywords: Dynamic fracture; Crack mechanics; Stress waves; Numerical methods

\footnotetext{
* Corresponding author.
}

0022-5096/00/\$ - see front matter (C) 2000 Elsevier Science Ltd. All rights reserved. PII: S0022-5096(99)00069-1 


\section{Introduction}

A crack propagating through a region of locally varying critical energy release rate in a 3D solid must speed up or slow down to accommodate those fluctuations. The resulting local crack accelerations produce elastic waves which either remain confined to a region close to the crack tip, or propagate outwards from the crack tip as body and surface waves which may intersect distant portions of the crack front. The confined waves, discovered in recent studies (Morrissey and Rice, 1996, 1998; Ramanathan and Fisher, 1997) of mode I crack propagation, are called crack front waves. In both cases, the waves cause portions of the crack front which are remote in space and time from the wave origin to alter velocity as well. Long-range interactions of the body-wave type, under sustained random fluctuations in critical fracture energy, were shown by Perrin and Rice (1994) to cause a crack in a model scalar elastic to become increasingly disordered. The type of elastic wave that remains local to the crack front, i.e., the crack front wave, can cause a mode I crack in a linear elastic solid to become disordered even more rapidly than suggested by that scalar analysis.

In this paper a methodology is developed to simulate mode I dynamic cracking within the singular crack model, based on first-order in-plane perturbations from crack propagation at uniform speed with a straight front. It is similar in form to that of Rice et al. (1994) and Perrin and Rice (1994), but for a true elastic solid rather than for a model scalar elastic solid as was considered in those works. This method is then applied to analyze crack front waves and resonances for single spatial modes in crack shape, as well as cracking through regions of randomly heterogeneous critical energy release rate.

This methodology begins with the perturbative solution for mode I dynamic cracking of Willis and Movchan (1995), as expressed in terms of energy release rate by Ramanathan and Fisher (1997). The latter formulation, given by its authors in the wavenumber-frequency domain, is inverted to the wavenumbertime domain to obtain an equation of motion for the mode I crack front which is similar in structure to that of Rice et al. (1994) for the scalar case.

In all of these methods, unlike the spectral method of Geubelle and Rice (1995) employed by Morrissey and Rice $(1996,1998)$ with a non-singular cohesive zone fracture model, crack motion is regarded as a linearized, in-plane perturbation in crack velocity about a constant velocity $v_{0}$, and the crack front is a mathematically sharp object sustaining the normal type of $1 / \sqrt{r}$ stress singularity. This allows a more accurate description of the crack front wave. Furthermore, the lower dimensionality of the system (1 less space dimension describing the crack) enables simulations to be performed on current workstations, unlike the method of Geubelle and Rice (1995) which usually requires supercomputing power for meaningful simulations.

The problem considered is that of a half-plane crack in an infinite solid, as depicted in Fig. 1. The crack is confined to the $x-z$ plane, and is traction-free along its faces. The rest value, $G_{\text {rest }}$, of the energy release rate $G$ is constant in configurations for which the crack remains straight as it propagates, taking the 
value $G_{\text {rest }}$ there. In general, $G=g(v) G_{\text {rest }}$, following the result of Freund (1972), where $g(v)$ is a universal function of crack velocity with the properties, $g\left(0^{+}\right)=1$, and $g\left(c_{\mathrm{R}}\right)=0, c_{\mathrm{R}}$ being the Rayleigh speed. In the absence of any spatial variation of critical energy release rate $G_{\text {crit }}$, the crack propagates in the positive $x$-direction with a uniform velocity $v_{0}$ satisfying $G_{\text {crit }}=g\left(v_{0}\right) G_{\text {rest }}$.

However, in a region with a deviation in critical fracture energy, $\Delta G_{\text {crit }}(z, x)$, from the background value $G_{\text {crito }}$ the velocity is perturbed, producing a deviation in crack shape $A(z, t)$ and also perturbing $G_{\text {rest }}$. Here $A(z, t)=a(z, t)-v_{0} t$ where the crack front lies along $x=a(z, t)$ in the $x, z$ plane. Perturbing Freund's result to first order, the crack propagates according to:

$$
\frac{\Delta G_{\text {crit }}\left(z, v_{0} t\right)}{G_{\text {crit }_{0}}}=\frac{g^{\prime}\left(v_{0}\right)}{g\left(v_{0}\right)} \frac{\partial A(z, t)}{\partial t}+\frac{\Delta G_{\text {rest }}\left[z, t ; A\left(z^{\prime}, t^{\prime}\right),-\infty<z^{\prime}<+\infty, t^{\prime} \leq t\right]}{G_{\text {rest }_{0}}}
$$

where the notation is to indicate $\Delta G_{\text {rest }}$ is a functional of $A(z, t)$, determined by the equations of elastodynamics. We will now develop an expression for the relationship between $\Delta G_{\text {crit }}(z, x)$ and $A(z, t)$ based on the work of Willis and Movchan (1995) and Ramanathan and Fisher (1997).

\section{Relationship between $\Delta G_{\text {crit }}(z, x)$ and $A(z, t)$}

The linear perturbation analysis of Ramanathan and Fisher (and that of Willis and Movchan) requires substituting $v_{0} t$ for $x$ in the evaluation of $\Delta G_{\text {crit }}(z, x)$. This allows relating the deviation in crack position $A(z, t)$ and $\Delta G$ by a linear functional, resulting in the equation of motion

$$
\frac{\Delta \hat{G}(k, \omega)}{G_{0}}=-\hat{P}(k, \omega) \hat{A}(k, \omega),
$$

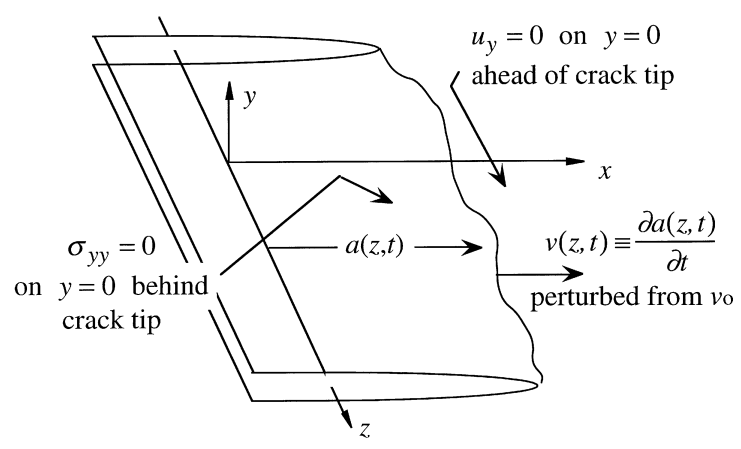

Fig. 1. Geometry of the fracture problem addressed. 
where

$$
\hat{A}(k, \omega)=\int_{-\infty}^{+\infty} \int_{-\infty}^{+\infty} \mathrm{e}^{-i k z-i \omega t} A(z, t) \mathrm{d} z \mathrm{~d} t
$$

and

$$
\Delta \hat{G}(k, \omega)=\int_{-\infty}^{+\infty} \int_{-\infty}^{+\infty} \mathrm{e}^{-i k z-i \omega t} \Delta G_{\text {crit }}\left(z, v_{0} t\right) \mathrm{d} z \mathrm{~d} t
$$

The function $\hat{P}(k, \omega)$ is given by Ramanathan and Fisher (1997) in the wavenumber-frequency domain:

$$
\begin{aligned}
\hat{P}(k, \omega)= & \frac{2 c_{\mathrm{R}}|k|}{c_{\mathrm{R}}^{2}-v_{0}^{2}} \sqrt{c_{\mathrm{R}}^{2}-H}-\frac{c_{\mathrm{d}}|k|}{c_{\mathrm{d}}^{2}-v_{0}^{2}} \sqrt{c_{\mathrm{d}}^{2}-H} \\
& -|k| \frac{1}{\pi} \int_{c_{\mathrm{s}}^{2}}^{c_{\mathrm{d}}^{2}} \tan ^{-1}\left(\frac{4 \sqrt{1-J / c_{\mathrm{d}}^{2}} \sqrt{J / c_{\mathrm{s}}^{2}-1}}{\left(2-J / c_{\mathrm{s}}^{2}\right)^{2}}\right) \frac{2 v_{0}^{2} J-H\left(J+v_{0}^{2}\right)}{\sqrt{J(J-H)}\left(J-v_{0}^{2}\right)^{2}} \mathrm{~d} J
\end{aligned}
$$

where $H=v_{0}^{2}+\omega^{2} / k^{2}$ and $c_{\mathrm{R}}, c_{\mathrm{s}}, c_{\mathrm{d}}$ are the Rayleigh, shear, and dilatational wave speeds, respectively. In Morrissey and Rice (1998), $\hat{P}(k, \omega)$ is written as $2 / \hat{H}(k, \omega)$, that transfer function $\hat{H}(k, \omega)$ being distinct from the $H$ defined above, and the expression for $\hat{H}(k, \omega)$ is given in terms of a function introduced by Willis and Movchan (1995). We note that $\hat{P}(k, \omega)$ has the form $|k|$ times a function of $\omega^{2} / k^{2}$, and is real for $\omega^{2} / k^{2}<c_{\mathrm{R}}^{2}-v_{0}^{2}$.

As shown in Fig. 2, and observed by Ramanathan and Fisher (1997), the function $\hat{P}(k, \omega)$ has a simple zero corresponding to a propagating mode of velocity $c_{\mathrm{f}}$, slightly less than the Rayleigh wave speed $c_{\mathrm{R}}$. Here $c_{\mathrm{f}}$ is the speed

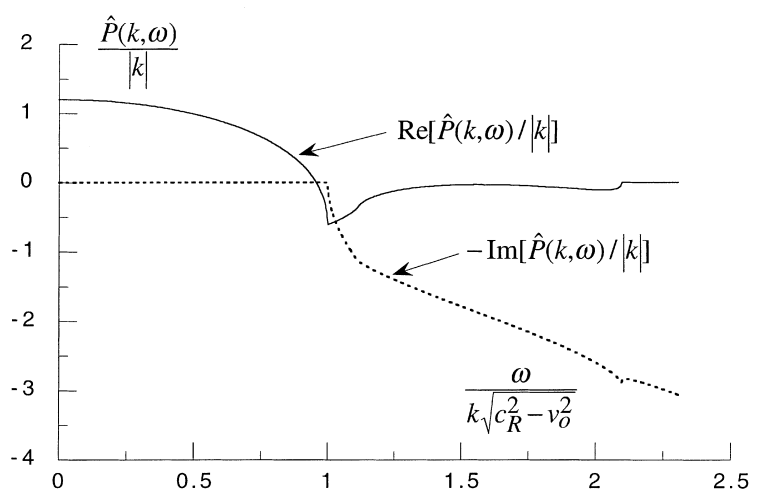

Fig. 2. Graph of the function $\hat{P}(k, \omega)$ for Poisson ratio 0.25 and $v_{0}=0.5 c_{\mathrm{R}}$. Note the simple root corresponding to the crack front wave. 
relative to a fixed point on the fracture plane at which the wave was nucleated or through which it passed, simultaneously with the crack front, in earlier crack motion. The velocity $c_{\mathrm{f}}$ varies with the unperturbed crack velocity $v_{0}$, approaching the Rayleigh wave speed with increasing crack speed, as shown in Fig. 3(a). The speed $c$ of the propagating mode parallel to the crack front may be related to $c_{\mathrm{f}}$ by the Pythagorean rule; if the wave has moved distance $c_{\mathrm{f}} t$ from such fixed position on the fracture plane as mentioned above, and has moved by $c t$ parallel to the crack front, during a time in which the front itself has advanced by $v_{0} t$, then $\left(c_{\mathrm{f}} t\right)^{2}=(c t)^{2}+\left(v_{0} t\right)^{2}$, and thus $c=\sqrt{c_{\mathrm{f}}^{2}-v_{0}^{2}}$. In fact, the $\omega / k \sqrt{c_{\mathrm{R}}^{2}-v_{0}^{2}}$ axis position at the zero of $\hat{P}(k, \omega)$ in Fig. 2 corresponds to $c / \sqrt{c_{\mathrm{R}}^{2}-v_{0}^{2}}$, and that measure of wave speed is shown as a function of $v_{0}$ in Fig. 3(b).

In order to use the result $\hat{P}(k, \omega)$ in simulations, the equation of motion (2a) must be partially inverted to the time domain.

\subsection{Inversion of the equation of motion}

We make the substitution $\sqrt{J}=\eta$ in Eq. (3) and define

$$
\Theta\left(\eta ; c_{\mathrm{d}}, c_{\mathrm{s}}\right)=\frac{2}{\pi} \tan ^{-1}\left(\frac{4 \sqrt{1-\eta^{2} / c_{\mathrm{d}}^{2}} \sqrt{\eta^{2} / c_{\mathrm{s}}^{2}-1}}{\left(2-\eta^{2} / c_{\mathrm{s}}^{2}\right)^{2}}\right),
$$

which takes on values from 0 to 1 over the range of the integral, to obtain

$$
\begin{aligned}
\hat{P}(k, \omega)= & \frac{2 c_{\mathrm{R}}}{c_{\mathrm{R}}^{2}-v_{0}^{2}} \sqrt{\left(c_{\mathrm{R}}^{2}-v_{0}^{2}\right) k^{2}-\omega^{2}}-\frac{c_{\mathrm{d}}}{c_{\mathrm{d}}^{2}-v_{0}^{2}} \sqrt{\left(c_{\mathrm{d}}^{2}-v_{0}^{2}\right) k^{2}-\omega^{2}} \\
& -\int_{c_{\mathrm{s}}}^{c_{\mathrm{d}}} \Theta\left(\eta ; c_{\mathrm{d}}, c_{\mathrm{s}}\right) \frac{v_{0}^{2} k^{2}}{\left(\eta^{2}-v_{0}^{2}\right)} \frac{\mathrm{d} \eta}{\sqrt{\left(\eta^{2}-v_{0}^{2}\right) k^{2}-\omega^{2}}} \\
& +\omega^{2} \int_{c_{\mathrm{s}}}^{c_{\mathrm{d}}} \Theta\left(\eta ; c_{\mathrm{d}}, c_{\mathrm{s}}\right) \frac{\left(\eta^{2}+v_{0}^{2}\right)}{\left(\eta^{2}-v_{0}^{2}\right)^{2}} \frac{\mathrm{d} \eta}{\sqrt{\left(\eta^{2}-v_{0}^{2}\right) k^{2}-\omega^{2}}}
\end{aligned}
$$

The branches of the square root are chosen consistently with our definition of the transform in Eq. (2), with use of $-i \omega$ rather than $+i \omega$ as in Ramanathan and Fisher (1997), such that

$$
\sqrt{p^{2}-\omega^{2}}=i \operatorname{sign}(\omega) \sqrt{\omega^{2}-p^{2}} \text { for large real } \omega,
$$

which implies the remaining term involving $\omega$ in the integrands above is

$$
\frac{1}{\sqrt{\left(\eta^{2}-v_{0}^{2}\right) k^{2}-\omega^{2}}} \text { for } \omega^{2}<\left(\eta^{2}-v_{0}^{2}\right) k^{2},
$$




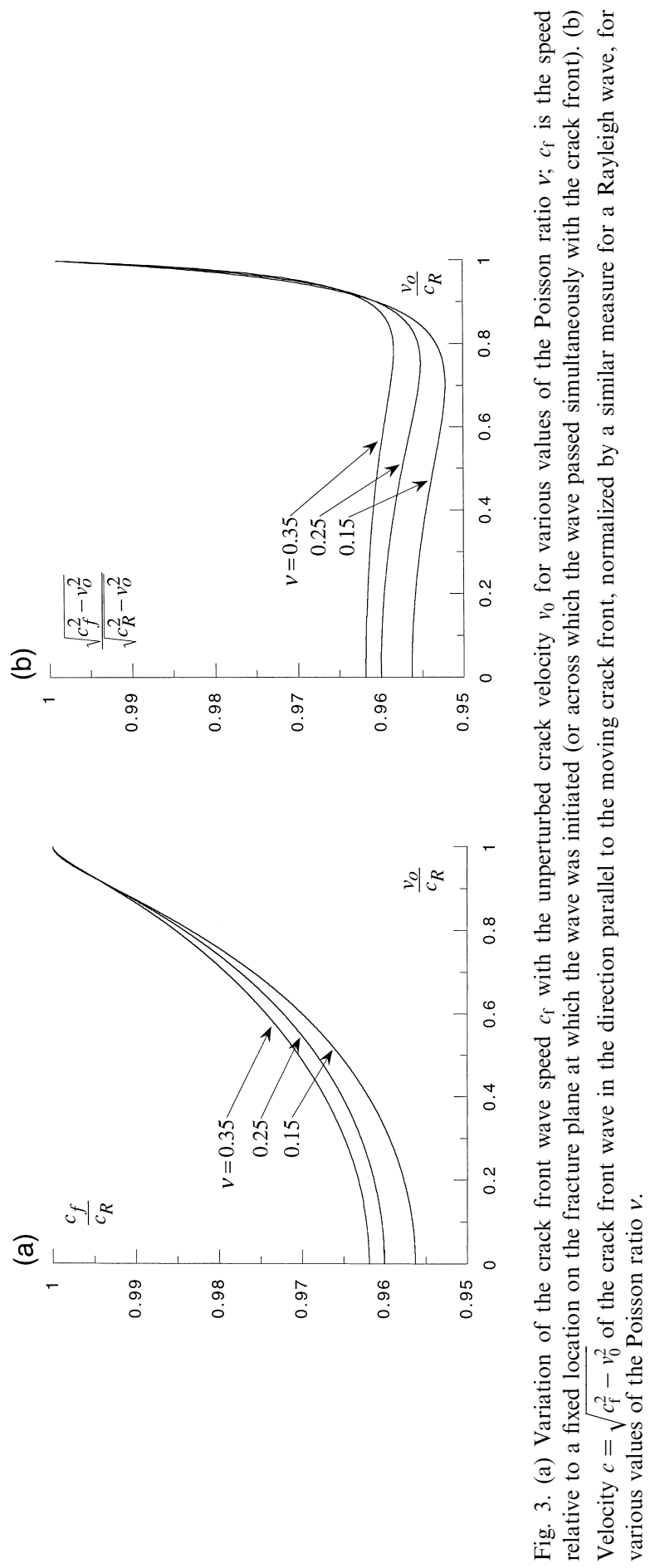


and

$$
\frac{-i \operatorname{sign}(\omega)}{\sqrt{\omega^{2}-\left(\eta^{2}-v_{0}^{2}\right) k^{2}}} \text { for } \omega^{2}>\left(\eta^{2}-v_{0}^{2}\right) k^{2} .
$$

That is recognized as the Fourier transform of $H(t) J_{0}\left(\sqrt{\eta^{2}-v_{0}^{2}}|k| t\right)$, where $H(t)$ is the Heaviside unit step function, and $J_{0}$ is the Bessel function of the first kind.

Henceforth, let us denote the timewise Fourier transform of a function $f(t)$ as $\operatorname{FT}[f(t)]$. We may then write

$$
\begin{aligned}
\hat{P}(k, \omega)= & \frac{2 c_{\mathrm{R}}}{c_{\mathrm{R}}^{2}-v_{0}^{2}} \sqrt{\left(c_{\mathrm{R}}^{2}-v_{0}^{2}\right) k^{2}-\omega^{2}}-\frac{c_{\mathrm{d}}}{c_{\mathrm{d}}^{2}-v_{0}^{2}} \sqrt{\left(c_{\mathrm{d}}^{2}-v_{0}^{2}\right) k^{2}-\omega^{2}} \\
& -\int_{c_{\mathrm{s}}}^{c_{\mathrm{d}}} \Theta\left(\eta ; c_{\mathrm{d}}, c_{\mathrm{s}}\right) \mathrm{FT}\left[H(t) J_{0}\left(\sqrt{\eta^{2}-v_{0}^{2}}|k| t\right)\right] \frac{v_{0}^{2} k^{2} \mathrm{~d} \eta}{\left(\eta^{2}-v_{0}^{2}\right)} \\
& +\omega^{2} \int_{c_{\mathrm{s}}}^{c_{\mathrm{d}}} \Theta\left(\eta ; c_{\mathrm{d}}, c_{\mathrm{s}}\right) \mathrm{FT}\left[H(t) J_{0}\left(\sqrt{\eta^{2}-v_{0}^{2}}|k| t\right)\right] \frac{\left(\eta^{2}+v_{0}^{2}\right) \mathrm{d} \eta}{\left(\eta^{2}-v_{0}^{2}\right)^{2}}
\end{aligned}
$$

In order to invert the first two terms of $\hat{P}(k, \omega)$, we extract their non-vanishing parts as $\omega \rightarrow \infty$ and thus note that

$$
\sqrt{p^{2}-\omega^{2}}=i \omega+\left(-i \omega+\sqrt{p^{2}-\omega^{2}}\right)=\mathrm{FT}\left[\frac{\partial}{\partial t}+\frac{p H(t) J_{1}(p t)}{t}\right],
$$

where $p^{2}$ will represent either $\left(c_{\mathrm{R}}^{2}-v_{0}^{2}\right) k^{2}$ or $\left(c_{\mathrm{d}}^{2}-v_{0}^{2}\right) k^{2}$.

Observing also that $\mathrm{FT}\left\{\omega^{2} \mathrm{FT}[f(t)]\right\}=-\mathrm{FT}\left[\mathrm{d}^{2} f(t) / \mathrm{d} t^{2}\right]$, and exchanging the inverse Fourier transform with the integral over $\eta$ in the expression for $\hat{P}(k, \omega)$, we may thus write

$$
\begin{aligned}
\hat{P}(k, t)= & \left(\frac{2 c_{\mathrm{R}}}{c_{\mathrm{R}}^{2}-v_{0}^{2}}-\frac{c_{\mathrm{d}}}{c_{\mathrm{d}}^{2}-v_{0}^{2}}\right) \frac{\partial}{\partial t}+2 c_{\mathrm{R}} k^{2} H(t) \frac{J_{1}\left(\sqrt{c_{\mathrm{R}}^{2}-v_{0}^{2}}|k| t\right)}{\sqrt{c_{\mathrm{R}}^{2}-v_{0}^{2}}|k| t} \\
& -c_{\mathrm{d}} k^{2} H(t) \frac{J_{1}\left(\sqrt{c_{\mathrm{d}}^{2}-v_{0}^{2}}|k| t\right)}{\sqrt{c_{\mathrm{d}}^{2}-v_{0}^{2}}|k| t} \\
& -\int_{c_{\mathrm{s}}}^{c_{\mathrm{d}}} \Theta\left(\eta ; c_{\mathrm{d}}, c_{\mathrm{s}}\right)\left[H(t) J_{0}\left(\sqrt{\eta^{2}-v_{0}^{2}}|k| t\right)\right] \frac{v_{0}^{2} k^{2} \mathrm{~d} \eta}{\left(\eta^{2}-v_{0}^{2}\right)} \\
& -\frac{\partial^{2}}{\partial t^{2}} \int_{c_{\mathrm{s}}}^{c_{\mathrm{d}}} \Theta\left(\eta ; c_{\mathrm{d}}, c_{\mathrm{s}}\right)\left[H(t) J_{0}\left(\sqrt{\eta^{2}-v_{0}^{2}}|k| t\right)\right] \frac{\left(\eta^{2}+v_{0}^{2}\right) \mathrm{d} \eta}{\left(\eta^{2}-v_{0}^{2}\right)^{2}}
\end{aligned}
$$


For simulations we will need to find the inverse of $-\hat{P}(k, \omega) \hat{A}(k, \omega)$, which is $-\hat{P}(k, t) \otimes \hat{A}(k, t)$, where $\otimes$ denotes convolution in time. The convolution yields

$$
\begin{aligned}
-\hat{P}(k, t) \otimes \hat{A}(k, t)= & \left(\frac{c_{\mathrm{d}}}{c_{\mathrm{d}}^{2}-v_{0}^{2}}-\frac{2 c_{\mathrm{R}}}{c_{\mathrm{R}}^{2}-v_{0}^{2}}\right) \frac{\partial \hat{A}(k, t)}{\partial t} \\
& -2 c_{\mathrm{R}} k^{2} \int_{-\infty}^{t} \frac{J_{1}\left(\sqrt{c_{\mathrm{R}}^{2}-v_{0}^{2}}|k|\left(t-t^{\prime}\right)\right)}{\sqrt{c_{\mathrm{R}}^{2}-v_{0}^{2}}|k|\left(t-t^{\prime}\right)} \hat{A}\left(k, t^{\prime}\right) \mathrm{d} t^{\prime} \\
& +c_{\mathrm{d}} k^{2} \int_{-\infty}^{t} \frac{J_{1}\left(\sqrt{c_{\mathrm{d}}^{2}-v_{0}^{2}}|k|\left(t-t^{\prime}\right)\right)}{\sqrt{c_{\mathrm{d}}^{2}-v_{0}^{2}}|k|\left(t-t^{\prime}\right)} \hat{A}\left(k, t^{\prime}\right) \mathrm{d} t^{\prime} \\
& +\int_{c_{\mathrm{s}}}^{c_{\mathrm{d}}} \int_{-\infty}^{t} \Theta\left(\eta ; c_{\mathrm{d}}, c_{\mathrm{s}}\right) \frac{v_{0}^{2}}{\left(\eta^{2}-v_{0}^{2}\right)} J_{0}\left(\sqrt{\eta^{2}-v_{0}^{2}}|k|\left(t-t^{\prime}\right)\right) \\
& \times \hat{A}\left(k, t^{\prime}\right) \mathrm{d} t^{\prime} \mathrm{d} \eta+\frac{\partial^{2}}{\partial t^{2}} \int_{c_{\mathrm{s}}}^{c_{\mathrm{d}}} \int_{-\infty}^{t} \Theta\left(\eta ; c_{\mathrm{d}}, c_{\mathrm{s}}\right) \frac{\left(\eta^{2}+v_{0}^{2}\right)}{\left(\eta^{2}-v_{0}^{2}\right)^{2}} \\
& \times J_{0}\left(\sqrt{\eta^{2}-v_{0}^{2}}|k|\left(t-t^{\prime}\right)\right) \hat{A}\left(k, t^{\prime}\right) \mathrm{d} t^{\prime} \mathrm{d} \eta
\end{aligned}
$$

Taking the second derivative with respect to time inside the integrand of the last term, and collecting like terms, we have one term that corresponds to the instantaneous dependence of energy release rate on crack motion, and other terms that carry the wave-mediated change in energy release rate generated by all prior nonuniformities of crack motion:

$$
\begin{aligned}
-\hat{P}(k, t) \otimes \hat{A}(k, t)= & {\left[\frac{c_{\mathrm{d}}}{c_{\mathrm{d}}^{2}-v_{0}^{2}}-\frac{2 c_{\mathrm{R}}}{c_{\mathrm{R}}^{2}-v_{0}^{2}}+\int_{c_{\mathrm{s}}}^{c_{\mathrm{d}}} \Theta\left(\eta ; c_{\mathrm{d}}, c_{\mathrm{s}}\right) \frac{\left(\eta^{2}+v_{0}^{2}\right) \mathrm{d} \eta}{\left(\eta^{2}-v_{0}^{2}\right)^{2}}\right] } \\
& \times \frac{\partial \hat{A}(k, t)}{\partial t}+k^{2} \int_{-\infty}^{t}\left[c_{\mathrm{d}} \frac{J_{1}\left(k \alpha_{\mathrm{d}} c_{\mathrm{d}}\left(t-t^{\prime}\right)\right)}{k \alpha_{\mathrm{d}} c_{\mathrm{d}}\left(t-t^{\prime}\right)}\right. \\
& \left.-2 c_{\mathrm{R}} \frac{J_{1}\left(k \alpha_{\mathrm{R}} c_{\mathrm{R}}\left(t-t^{\prime}\right)\right)}{k \alpha_{\mathrm{R}} c_{\mathrm{R}}\left(t-t^{\prime}\right)}\right] \hat{A}\left(k, t^{\prime}\right) \mathrm{d} t^{\prime} \\
& +\frac{k^{2}}{2} \int_{-\infty}^{t} \int_{c_{\mathrm{s}}}^{c_{\mathrm{d}}} \Theta\left(\eta ; c_{\mathrm{d}}, c_{\mathrm{s}}\right)\left[\frac{\eta^{2}+v_{0}^{2}}{\eta^{2}-v_{0}^{2}} J_{2}\left(k \alpha_{\eta} \eta\left(t-t^{\prime}\right)\right)\right. \\
& \left.-J_{0}\left(k \alpha_{\eta} \eta\left(t-t^{\prime}\right)\right)\right] \hat{A}\left(k, t^{\prime}\right) \mathrm{d} \eta \mathrm{d} t^{\prime}
\end{aligned}
$$


Here we have simplified notation by introducing the definition $\alpha_{\gamma}=\sqrt{1-v_{0}^{2} / c_{\gamma}^{2}}$ for any given wave speed $c_{\gamma}$ and unperturbed crack velocity $v_{0}$. Note that $\alpha_{\gamma} c_{\gamma}$ is the speed with which a wave of speed $c_{\gamma}$ relative to a fixed point on the fracture plane, nucleated as the crack passes through that point, travels in the direction parallel to the moving crack front. Also, $\alpha_{\eta}=\sqrt{1-v_{0}^{2} / \eta^{2}}$.

Lastly, we obtain the following form, for comparison with the analogous formula for a crack moving in a model scalar elastic solid derived by Rice et al. (1994):

$$
-\hat{P}(k, t) \otimes \hat{A}(k, t)=C_{v}\left(v_{0}\right) \frac{\partial \hat{A}(k, t)}{\partial t}+2 I(k, t)
$$

where

$$
I(k, t)=\int_{-\infty}^{t} \mathrm{~d} t^{\prime} B_{I}\left(k, t-t^{\prime}\right) \hat{A}\left(k, t^{\prime}\right) .
$$

The equation of motion is then written in the wavenumber-time domain as:

$$
\Delta \hat{G}_{\text {crit }}\left(k, v_{0} t\right)=G_{\text {crit }_{0}}\left(C_{v}\left(v_{0}\right) \frac{\partial \hat{A}(k, t)}{\partial t}+2 I(k, t)\right)
$$

For mode I the corresponding quantities are:

$$
C_{v}\left(v_{0}\right)=\frac{c_{\mathrm{d}}}{c_{\mathrm{d}}^{2}-v_{0}^{2}}-\frac{2 c_{\mathrm{R}}}{c_{\mathrm{R}}^{2}-v_{0}^{2}}+\int_{c_{\mathrm{s}}}^{c_{\mathrm{d}}} \Theta\left(\eta ; c_{\mathrm{d}}, c_{\mathrm{s}}\right) \frac{\left(\eta^{2}+v_{0}^{2}\right)}{\left(\eta^{2}-v_{0}^{2}\right)^{2}} \mathrm{~d} \eta
$$

and

$$
\begin{aligned}
B_{I}(k, t)= & \frac{k^{2}}{2}\left[c_{\mathrm{d}} \frac{J_{1}\left(k \alpha_{\mathrm{d}} c_{\mathrm{d}} t\right)}{k \alpha_{\mathrm{d}} c_{\mathrm{d}} t}-2 c_{\mathrm{R}} \frac{J_{1}\left(k \alpha_{\mathrm{R}} c_{\mathrm{R}} t\right)}{k \alpha_{\mathrm{R}} c_{\mathrm{R}} t}\right] \\
& +\frac{k^{2}}{4} \int_{c_{\mathrm{s}}}^{c_{\mathrm{d}}} \Theta\left(\eta ; c_{\mathrm{d}}, c_{\mathrm{s}}\right)\left[\frac{\eta^{2}+v_{0}^{2}}{\eta^{2}-v_{0}^{2}} J_{2}\left(k \alpha_{\eta} \eta t\right)-J_{0}\left(k \alpha_{\eta} \eta t\right)\right] \mathrm{d} \eta
\end{aligned}
$$

\subsection{Reduction to $2 D$ results}

Comparing to Eq. (1), the coefficient of instantaneous velocity in Eq. (12), $C_{v}\left(v_{0}\right)$, should in fact be equal to $g^{\prime}\left(v_{0}\right) / g\left(v_{0}\right)$, derivable from Freund (1972) for unsteady $2 \mathrm{D}$ crack motion. As discussed earlier, $g\left(v_{0}\right)$ is a universal function of crack velocity that describes the reduction in energy flow to the crack tip with crack speed.

From Freund (1972), $g\left(v_{0}\right)=\left[\hat{k}\left(v_{0}\right)\right]^{2} f\left(v_{0}\right)$, where (see also Morrissey and Rice, 1998) 


$$
\hat{k}\left(v_{0}\right)=\frac{1-v_{0} / c_{\mathrm{R}}}{\sqrt{1-v_{0} / c_{\mathrm{d}}} S_{+}\left(1 / v_{0}\right)} \text { and } f\left(v_{0}\right)=\frac{v_{0}^{2} \sqrt{1-v_{0}^{2} / c_{\mathrm{d}}^{2}}}{c_{\mathrm{s}}^{2}\left(1-v_{0}\right) r\left(v_{0}\right)}
$$

The function $r\left(v_{0}\right)$ is the Rayleigh function, defined by

$$
r\left(v_{0}\right)=4 \sqrt{1-v_{0}^{2} / c_{\mathrm{s}}^{2}} \sqrt{1-v_{0}^{2} / c_{\mathrm{d}}^{2}}-\left(2-v_{0}^{2} / c_{\mathrm{s}}^{2}\right)^{2}
$$

and $S_{+}\left(1 / v_{0}\right)$, arising from a Wiener-Hopf factorization in the solution of the dynamic $2 \mathrm{D}$ mode I crack problem, is

$$
S_{+}\left(1 / v_{0}\right)=\exp \left\{-\frac{v_{0}}{2} \int_{c_{\mathrm{s}}}^{c_{\mathrm{d}}} \Theta\left(\eta ; c_{\mathrm{d}}, c_{\mathrm{s}}\right) \frac{\mathrm{d} \eta}{\eta\left(\eta-v_{0}\right)}\right\} .
$$

We may then write

$$
\begin{aligned}
\frac{g^{\prime}\left(v_{0}\right)}{g\left(v_{0}\right)} & =\frac{f^{\prime}\left(v_{0}\right)}{f\left(v_{0}\right)}+2 \frac{k^{\prime}\left(v_{0}\right)}{k\left(v_{0}\right)} \\
& =\frac{2}{v_{0}}+\frac{c_{\mathrm{d}}}{c_{\mathrm{d}}^{2}-v_{0}^{2}}-\frac{2}{c_{\mathrm{R}}-v_{0}}-\frac{r^{\prime}\left(v_{0}\right)}{r\left(v_{0}\right)}+\int_{c_{\mathrm{s}}}^{c_{\mathrm{d}}} \Theta\left(\eta ; c_{\mathrm{d}}, c_{\mathrm{s}}\right) \frac{\mathrm{d} \eta}{\left(\eta-v_{0}\right)^{2}}
\end{aligned}
$$

At first glance, this looks little like the form derived for $C_{v}\left(v_{0}\right)$ in Eq. (13a), but proof of their equality is given in Appendix A. The proof involves reforming the term $r^{\prime}(v) / r(v)$ into a contour integral.

\section{Numerical formulation}

The numerical solution of the equations of motion (Eqs. (12) and (13)) begins with a discretization of the space dimension $(z)$ by $N$ FFT sample points with a spacing of $\Delta z$. Time is likewise discretized into discrete time intervals $\Delta t$, chosen small enough that the argument of the space-time kernel $B_{I}(k, t), k \alpha_{\mathrm{R}} c_{\mathrm{R}} t$, changes by a small fraction of $\pi$ with each increment of time $\Delta t$. We represent the fracture energy fluctuation by the discrete Fourier sum

$$
\Delta G_{\text {crit }}(z, x)=\sum_{n=-N / 2}^{N / 2} G_{n}(x) \mathrm{e}^{2 \pi i n z / \lambda_{z}}
$$

where $N$ is an even integer and the distribution is replicated periodically in the $z$ direction over length $\lambda_{z}$. The FFT may be used to find the $G_{n}$, given $\Delta G_{\text {crit }}$ at values of $z$ corresponding to the FFT sample points.

The crack position perturbation $A(z, t)$ and prior history functional $I(z, t)$ then have the representations 


$$
A(z, t)=\sum_{n=-N / 2}^{N / 2} A_{n}(t) \mathrm{e}^{2 \pi i n z / \lambda_{z}}, \quad I(z, t)=\sum_{n=-N / 2}^{N / 2} I_{n}(t) \mathrm{e}^{2 \pi i n z / \lambda_{z}}
$$

and the equation of motion is then

$$
G_{n}\left(v_{0} t\right)=G_{\text {crit }_{0}}\left(C_{v}\left(v_{0}\right) \frac{\mathrm{d} A_{n}(t)}{\mathrm{d} t}+2 I_{n}(t)\right)
$$

where

$$
I_{n}(t)=\int_{-\infty}^{t} B_{I}\left(k, t-t^{\prime}\right) A_{n}\left(t^{\prime}\right) \mathrm{d} t^{\prime}
$$

with $k=2 \pi n / \lambda_{z}$.

We assume that $A(z, t)$ has been calculated up to the present time, and that $A_{n}(t)$ and $B_{I}(k, t)$ are stored at discrete time intervals $\Delta t$. At each time step, the following loop is performed:

1. Calculate the $I_{n}(t)$ by convolving the $B_{I}(k, t)$ with the $A_{n}(t)$. Each convolution is done as a trapezoidal-rule sum based on values of the integrand at the discrete times.

2. Given the $G_{n}\left(v_{0} t\right)$ and $I_{n}(t)$, solve for the $\mathrm{d} A_{n}(t) / \mathrm{d} t$, and update to time $t+\Delta t$ by the Euler scheme $A_{n}(t+\Delta t)=A_{n}(t)+\Delta t \mathrm{~d} A_{n}(t) / \mathrm{d} t$.

3. Use the inverse FFT to find the updated $A(z, t+\Delta t)$ at the FFT sample points $z$.

4. Return to step (1) with $t$ replaced by $t+\Delta t$.

Each analysis begins with a crack moving steadily through a homogenous material in the positive $x$-direction. At some point (typically chosen to coincide with time $t=0$ ), the crack reaches an inhomogeneity, and $\Delta G_{\text {crit }}$ becomes non-zero. This produces a non-zero $\partial A(z, t) / \partial t$ in step (2), and hence a non-zero $A(z, t)$. Upon returning to step (1), the history functional $I_{n}(t)$ typically becomes non-zero. However, the zero in the function $\hat{P}(k, \omega)$ implies that there are motions of the crack front that produce no change in energy release rate. That is, there are motions $A_{n}(t)$, as crack front waves, that continue for all time even when $G_{n}(t)$ has returned to 0 .

\section{Simulations of dynamic crack growth}

\subsection{Modal analysis}

To see the effect of the crack front wave for mode I cracks, it is useful to first examine the response to a heterogeneity composed of a single spatial frequency, or

$$
\Delta G_{\text {crit }}\left(z, v_{0} t\right)=G_{k}(t) \cos (k z) .
$$


(Here the subscript $k$ denotes wavenumber, not index on the Fourier sum.) By linearity, the deviation in crack shape $A(z, t)$ is then

$$
A(z, t)=A_{k}(t) \cos (k z)
$$

One of the simplest choices for $G_{k}(t)$ is a Heaviside step, or

$$
G_{k}(t)=g_{k} G_{\text {crit }_{0}} H(t)
$$

where $g_{k}$ is the amplitude of the fluctuation in critical energy release rate as a fraction of the background value $G_{\text {crit }}$. Since $H(t)$ has non-vanishing Fourier transform at all frequencies, it will be non-zero near, $\omega^{2}=k^{2}\left(c_{\mathrm{f}}^{2}-v_{0}^{2}\right)$ the frequency at which $\hat{P}(k, \omega)$ has a simple zero, and its inverse has a simple pole. Therefore, it is expected that the time variation of the deviation in crack shape, $A_{k}(t)$, will ultimately be sinusoidal, representing the superposition of two propagating waves, one traveling to the left, and the other traveling to the right. The numerical simulation of this process is shown in Fig. 4. Except for the absence of the FFT, the procedure is identical to the outline of Section 3, with time step $\Delta t$ chosen such that $k \alpha_{\mathrm{f}} c_{\mathrm{f}} \Delta t=\pi / 10$.

Fig. 4 shows that, except for a short-term transient, each Fourier mode of crack deviation essentially behaves like a simple harmonic oscillator in which $G_{k}(t)$ plays the role of the driving force. Like the simple harmonic oscillator, if the driving force oscillates at the natural or resonant frequency, the response grows secularly. Thus, if $G_{k}(t)$ is of the form

$$
G_{k}(t)=g_{k} G_{\mathrm{crit}_{0}} \cos \left(k t \sqrt{c_{\mathrm{f}}^{2}-v_{0}^{2}}\right) H(t),
$$

we expect $A_{k}(t)$ to grow at long time as:

$$
A_{k}(t) \rightarrow \text { constant } \times k t \sqrt{c_{\mathrm{f}}^{2}-v_{0}^{2}} \times \cos \left(k t \sqrt{c_{\mathrm{f}}^{2}-v_{0}^{2}}+\varphi\right),
$$

where $c_{\mathrm{f}}$ is the crack front wave speed. The numerical result of this process is shown in Fig. 5.

The implication of these results for crack disordering, as discussed in Morrissey and Rice (1998) (in which it is now evident that their linear response function $L(k, t)$ is indeed ultimately sinusoidal), is that under a general, sustained random fluctuation of $\Delta G_{\text {crit }}$, the variances of crack shape and velocity grow linearly with time of propagation into the disordered region.

\subsection{Crack propagation through random $\Delta G_{\text {crit }}$}

Consider now a normal distribution of $\Delta G_{\text {crit }}(z, x)$ with two-point autocorrelation function $R(\Delta z, \Delta x)$. We will model $\Delta G_{\text {crit }}(z, x)$ as a random Fourier sum. Thus, 


$$
\Delta G_{\text {crit }}(z, x)=\sum_{n=-N / 2}^{N / 2} \sum_{m=-M / 2}^{M / 2} G_{n m} \mathrm{e}^{2 \pi i n x / \lambda_{x}} \mathrm{e}^{2 \pi i m z / \lambda_{z}}
$$

where $G_{00}$ is a normally-distributed real random variable of zero mean, where the other $G_{n m}$ are normally-distributed complex random variables of zero mean and uniformly distributed phase (thus assuring statistically independent real and imaginary parts), where $G_{(-n)(-m)}=\bar{G}_{n m}$ as required for a real-valued function, but where the real and imaginary parts of every other $G_{q p}$ are statistically independent of those of $G_{m n}$ whenever the ordered pair $q p$ differs from $n m$. Under these conditions it is readily seen that the ensemble average $\left\langle\Delta G_{\text {crit }}(z+\Delta z\right.$, $\left.x+\Delta x) \Delta G_{\text {crit }}(z, x)\right\rangle$ is independent of location $x, z$ and defines an auto-correlation function

$$
R(\Delta z, \Delta x)=\sum_{n=-N / 2}^{N / 2} \sum_{m=-M / 2}^{M / 2}\left\langle\left|G_{n m}\right|^{2}\right\rangle \mathrm{e}^{2 \pi i n \Delta z / \lambda_{z}} \mathrm{e}^{2 \pi i m \Delta x / \lambda_{x}} .
$$

The $R(\Delta z, \Delta x)$ for such a process is necessarily spatially periodic, since each realization of $\Delta G_{\text {crit }}(z, x)$ is. However, we can make $R(\Delta z, \Delta x)$, agree over the region $\left[-\lambda_{z} / 2<\Delta z<\lambda_{z} / 2,-\lambda_{x} / 2<\Delta x<\lambda_{x} / 2\right]$, within limitations of truncation of the Fourier series, with some given correlation function for a non-periodic process that has been generated as the Fourier transform of a non-negative twodimensional power spectral density. Then, in terms of the given $R(\Delta z, \Delta x)$, we have, by inversion of the series,

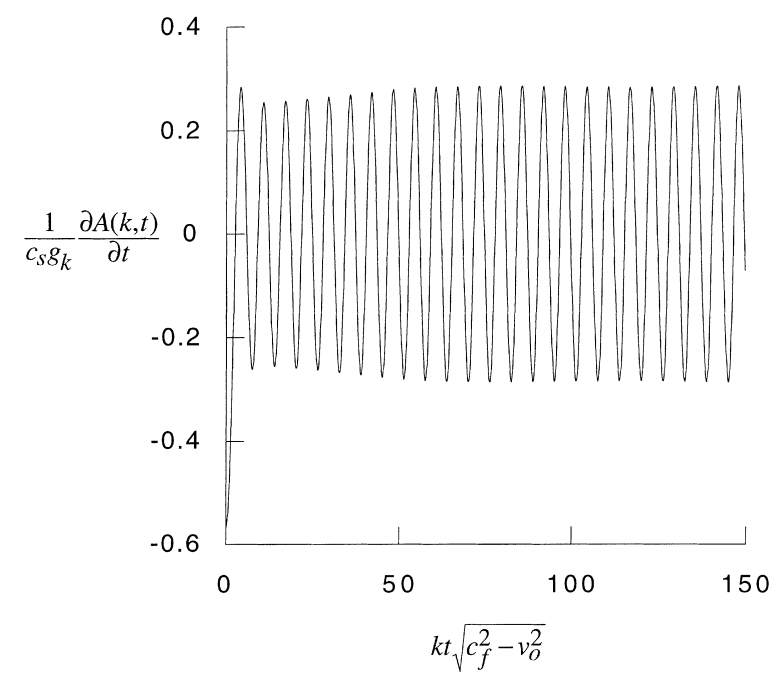

Fig. 4. Response of a single Fourier mode in crack shape to a Heaviside step in time of $\Delta G_{\text {crit }}$ in that mode. The sustained sinusoidal nature is indicative of a superposition of crack front waves, forming a standing mode. 


$$
\left\langle\left|G_{n m}\right|^{2}\right\rangle=\frac{1}{\lambda_{z} \lambda_{x}} \int_{-\lambda_{z} / 2}^{\lambda_{z} / 2} \int_{-\lambda_{x} / 2}^{\lambda_{x} / 2} R(\Delta z, \Delta x) \mathrm{e}^{-2 \pi i n \Delta x / \lambda_{x}} \mathrm{e}^{-2 \pi i m \Delta z / \lambda_{z}} \mathrm{~d} \Delta x \mathrm{~d} \Delta z
$$

In what follows, $R(\Delta z, \Delta x)$ is taken as $\sigma^{2} \mathrm{e}^{-\sqrt{\Delta x^{2}}}+\Delta z^{2} / b$ with correlation length $b$ equal to $8 \lambda_{z} / 512\left(\lambda_{z}\right.$ being the spatial period along $z$ ) or eight FFT sample spaces long. The standard deviation $\sigma$ is $0.25 G_{\text {crit }_{0}}$. However, to avoid negative values of $G_{\text {crit }}, \Delta G_{\text {crit }}(z, x), \quad$ is restricted to the range $[-3 \sigma,+3 \sigma]$, or $\left[-0.75 G_{\text {crit }},+0.75 G_{\text {crit }}\right]$. Since $95 \%$ of a normal distribution lies within three standard deviations of the mean, this is actually a small correction.

Fig. 6 shows the crack position $a(z, t)$ at various increasing times for a crack moving through a particular realization of the random $\Delta G_{\text {crit }}$ distribution described above. The X's show points at which the predicted perturbation has become so large that the local crack velocity is less than or equal to zero. (While one could, by fiat, demand that the crack velocity be constrained to the range $\left[0, c_{R}\right]$, the linearized formulation is strictly followed here.) They appear to correlate along lines with a slope determined by the crack front wave speed $c_{\mathrm{f}}$, denoted by dotted lines, implying that these "arrests" are the result of dynamics rather than purely the result of local regions of high critical energy release rate. The unperturbed crack speed $v_{0}$ was taken to be $0.5 c_{\mathrm{R}}$ for which $g\left(v_{0}\right)=0.525$, with Poisson ratio 0.25 , meaning that the rest value of $G$ (the value to which $G$ would revert if the crack were to suddenly stop) is about $2 G_{\text {crit }}$. But the maximum value of $G_{\text {crit }}$ is $1.75 G_{\text {crit }_{0}}$. Then it follows that without the assistance of elastic waves to reduce the local energy flow to the crack tip, the crack would always be

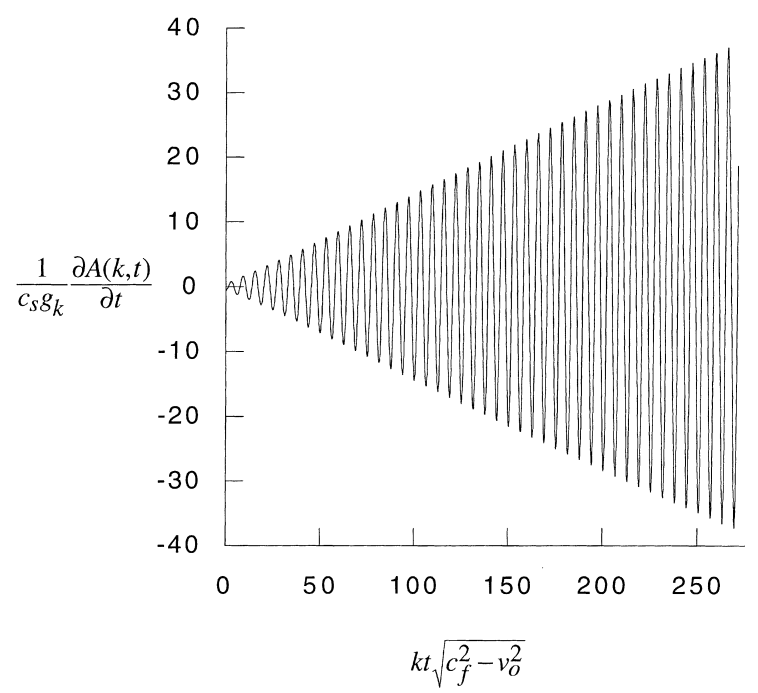

Fig. 5. Secular growth of a single Fourier mode in crack velocity, caused by a sinusoidal variation, with propagation distance, of $\Delta G_{\text {crit }}$ in that mode, with temporal frequency equal to the resonant frequency for that mode. 
everywhere sufficiently overloaded to cause fracture, and would therefore, never locally arrest.

However, there is no impression in Fig. 6 of increasing disorder or waviness in the crack shape with increasing growth through the disordered zone, which is a provable property of the analytical solution devised. A possible explanation can be found in a careful comparison of the discrete, simulated system to the continuous one.

In the continuous system, the heterogeneity in critical energy release rate $\Delta G_{\text {crit }}(z, x)=\Delta G_{\text {crit }}\left(z, v_{0} t\right)$ has spectral content at all frequencies $\omega$. But in the discrete system where $\Delta G_{\text {crit }}(z, x)$ is modeled by a random Fourier sum, the set of frequencies with non-vanishing spectral content is limited to $\omega_{n}=k_{x}^{n} v_{0}=2 \pi n v_{0} / \lambda_{x}, 0 \leq n \leq N / 2$. These frequencies may or may not coincide with the resonant frequencies for $A_{m}(t)$, the set of spatial Fourier modes describing the crack shape. Each mode in crack shape $A_{m}(t)$ is associated with a particular spatial wavenumber $k_{z}^{m}=2 \pi m / \lambda_{z}$, which resonates at a precise

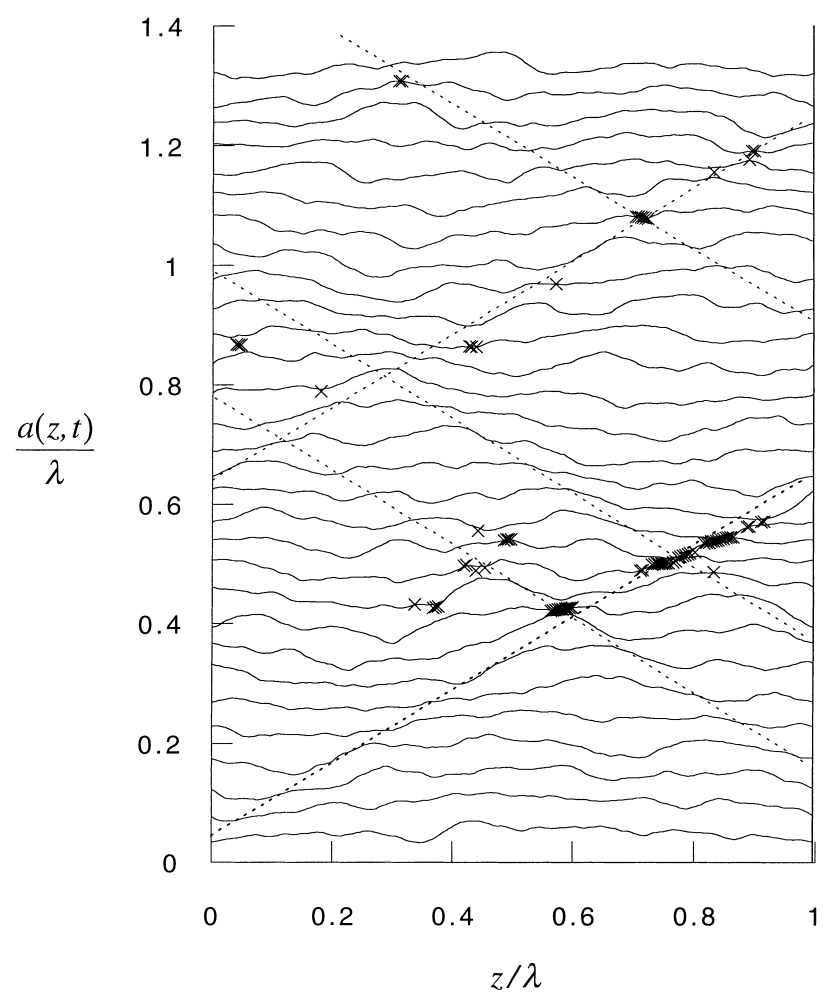

Fig. 6. Crack growth through a heterogeneous region modeled by random Fourier series. The X's mark places where the crack velocity fluctuation (within the linearized perturbations studied) became so severe as to make the net velocity $\leq 0$. These "arrest" events are a result of mode I dynamics. 
frequency $\omega_{m}=k_{z}^{m} \sqrt{c_{\mathrm{f}}^{2}-v_{0}^{2}}$. Thus, the $m$ th mode of crack shape can grow unboundedly if and only if, for some $n \leq N / 2, \omega_{n}=\omega_{m}$.

Fig. 7 shows an example of the resonant frequencies versus the available time frequencies for the Fourier sum representation of $\Delta G_{\text {crit }}$. Resonance occurs when a ' +' mark lies along the line where the two frequencies are equal. There is in fact no resonance in this figure, though some modes of crack shape come very close to resonating. It is possible to choose a set of spatial frequencies for the $\Delta G_{\text {crit }}$ distribution such that most or even all modes of crack shape $A_{m}(t)$ will resonate.

An example of crack propagation under such a condition is shown in Fig. 8. The only difference between the simulation performed for Fig. 8 and that of Fig. 6 is that in the former the fundamental wavelength $\lambda_{x}$ of $\Delta G_{\text {crit }}$ was shortened by $10 \%$, bringing the lower $2 / 3$ of crack shape modes into resonance. (Frequencies high enough to resonate the top third of spatial modes simply were not present in this analysis.) The deviation in crack shape in this case is decidedly larger, and there is a veritable forest of local arrest events - so many, in fact that the unaided eye can easily follow the tracks of the propagating crack front waves.

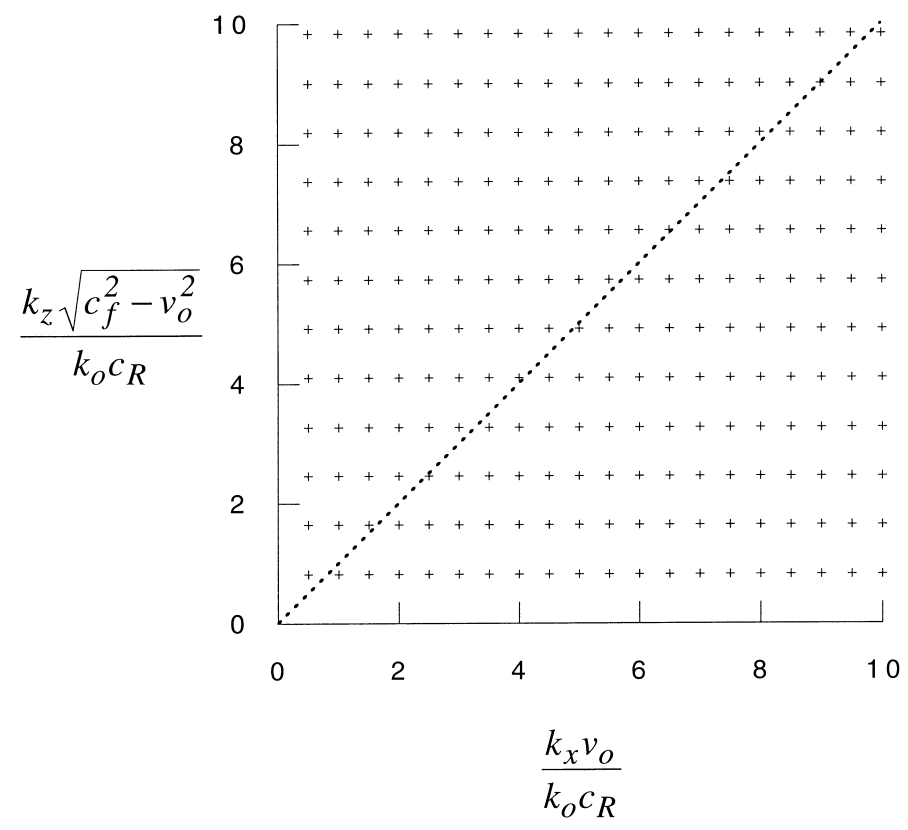

Fig. 7. Graph of resonant frequencies of the spatial modes of the crack front vs. the time-frequencies available in the representation of $\Delta G_{\text {crit }}$ by a random Fourier series. Resonance occurs only when a plus $(+)$ sign lies on the dotted line where the two frequencies are equal. 


\subsection{Modal analysis of crack propagation through random $\Delta G_{\text {crit }}$}

In order to reproduce the analytic prediction of linear growth of disorder in the propagating mode I crack front, it is clear that the spectrum of time frequencies in the representation of $\Delta G_{\text {crit }}\left(z, v_{0} t\right)$ must not be restricted as they are when the heterogeneity is modeled by a random Fourier sum. We will now re-examine the case of Section 4.1 where the heterogeneity $\Delta G_{\text {crit }}\left(z, v_{0} t\right)$ is composed of a single spatial component. Thus,

$$
\Delta G_{\text {crit }}\left(z, v_{0} t\right)=G_{k}(t) \cos (k z) \quad \text { and } \quad A(z, t)=A_{k}(t) \cos (k z) .
$$

But now $G_{k}(t)$ will vary randomly with time. In contrast to Section 4.2 , the

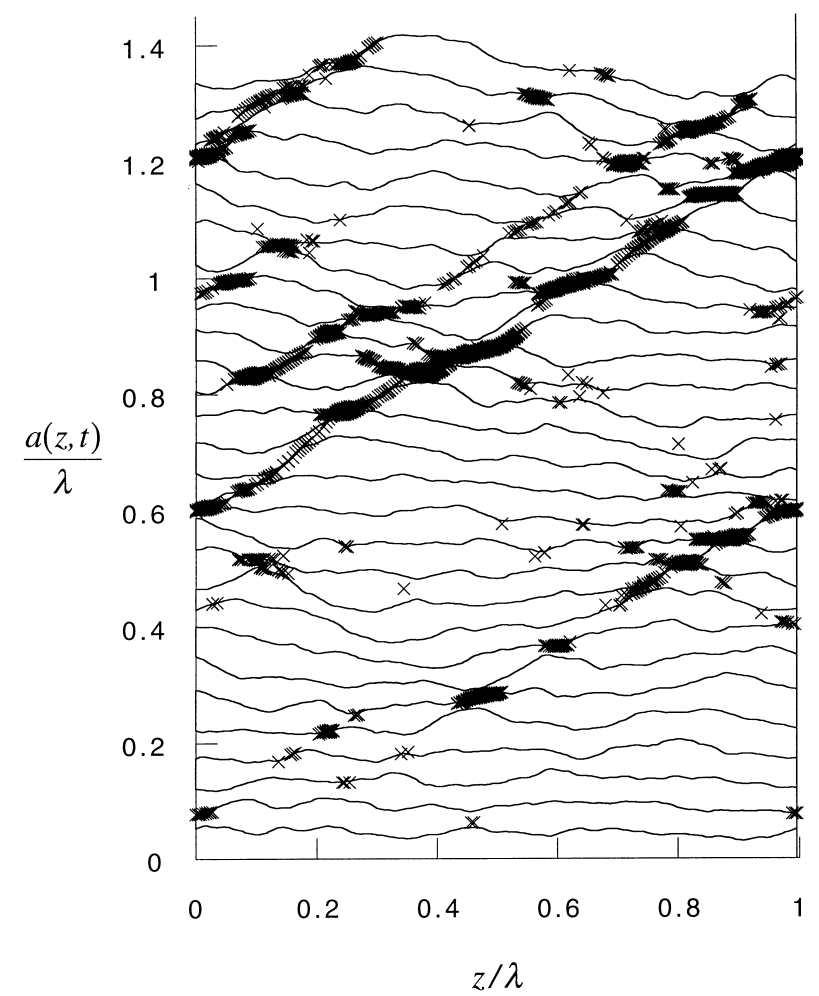

Fig. 8. Crack growth through a heterogeneous region modeled by random Fourier series. Wavelengths of the series in the direction of propagation were chosen to obtain resonance. Many more "arrest" events, marked by X's, are seen than in Fig. 6. 
random variation of $G_{k}(t)$ will now be evaluated on-the-fly, and will not be periodic in time.

Let $g_{\text {ran }}^{n}, \quad n=(0,1,2, \ldots)$, denote a sequence of normally-distributed, independent, random real numbers of zero mean and variance $\sigma_{g}^{2}$. An exponentially correlated sequence $g_{k}^{n}$ can be constructed from this one by the initial condition $g_{k}^{0}=g_{\text {ran }}^{0}$ combined with the recursion rule

$$
g_{k}^{n}=w_{1} g_{k}^{n-1}+w_{2} g_{\mathrm{ran}}^{n},
$$

where $w_{1}$ and $w_{2}$ are positive reals less than 1 . If $w_{1}^{2}+w_{2}^{2}=1$, the sequence $g_{k}^{n}$ is stationary with expected square modulus

$$
\left\langle\left(g_{k}^{n}\right)^{2}\right\rangle=\left\langle\left(g_{\text {ran }}^{n}\right)^{2}\right\rangle \equiv \sigma_{g}^{2} .
$$

The correlation of the sequence then follows the geometric rule

$$
\left\langle g_{k}^{n} g_{k}^{m}\right\rangle=w_{1}^{|n-m|} \sigma_{g}^{2} .
$$

If we now make the association $G_{k}(t)=G_{k}(n \Delta t)=g_{k}^{n} G_{\text {crit }}$, where $\Delta t$ is the time step of a simulation, then the autocorrelation of $G_{k}(t), R(\tau)$, is

$$
R(\tau)=\left\langle G_{k}(t) G_{k}(t+\tau)\right\rangle=w_{1}^{|\tau| / \Delta t} \sigma_{g}^{2} G_{\text {crit }_{0}}^{2} .
$$

Since the modal crack shape $A_{k}(t)$ resonates at frequency $k \alpha_{\mathrm{f}} c_{\mathrm{f}}$, an auspicious choice for $R(\tau)$, i.e. one which ensures good spectral content near the resonant frequency is

$$
R(\tau)=G_{\text {crit }}^{2} \sigma_{g}^{2} \mathrm{e}^{-k \alpha_{\mathrm{f}} c_{\mathrm{f}}|\tau|}
$$

or

$$
w_{1}=\mathrm{e}^{-k \alpha_{\mathrm{f}} c_{\mathrm{f}} \Delta t} .
$$

Alternatively, if we wish to model an exponentially correlated heterogeneity with spatial correlation length $b$ in the direction of crack propagation, i.e. $R(\tau)=G_{\text {crit }}^{2} \sigma_{g}^{2} \mathrm{e}^{-v_{0}|\tau| / b}$, then the corresponding choice for $w_{1}$ is $w_{1}=\mathrm{e}^{-v_{0} \Delta t / b}$.

Note that in principle, generating the values of $G_{k}(t)$ on-the-fly in this way produces spectral content at all time frequencies $\omega$ that are rational fractions of the temporal Nyquist frequency of the simulation, $\pi / \Delta t$. Therefore, assuming $k \alpha_{\mathrm{f}} c_{\mathrm{f}}<\pi / \Delta t$, the resonant frequency will be captured. (If $k \alpha_{\mathrm{f}} c_{\mathrm{f}}>\pi / \Delta t$, no resonance or growth of disorder can occur for the mode of crack shape with wavenumber $k$.)

Fig. 9 shows the expected value of the squared-amplitude of the deviation in crack shape $A_{k}(t)$ as a function of time, for crack growth through the modal heterogeneous toughness described above. The expected values were obtained from an ensemble average of 100 such simulations. In each simulation the unperturbed crack velocity $v_{0}$ was one-half of the Rayleigh wave speed, and the 


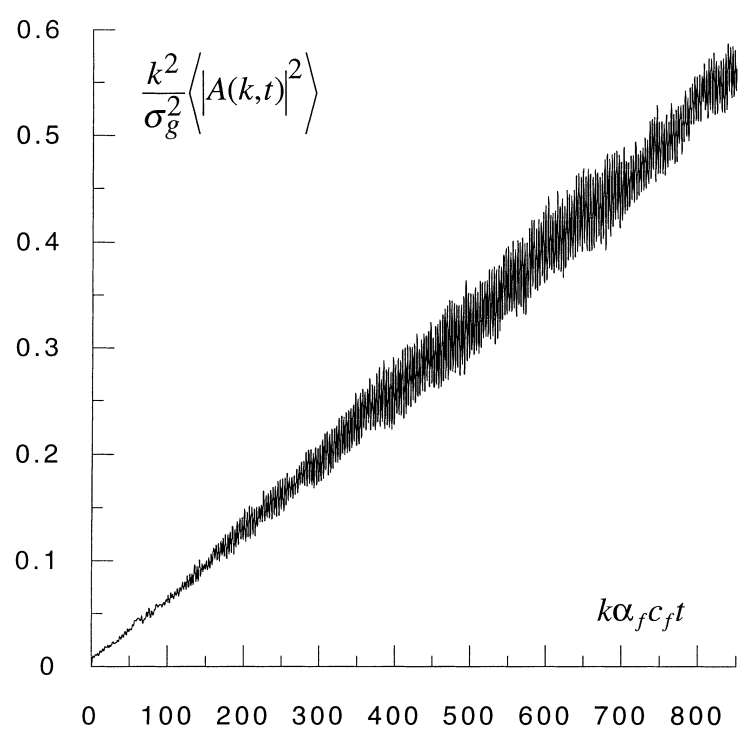

Fig. 9. Expected squared modulus of a single Fourier mode in crack shape, for a crack front propagating through a zone of randomly variable fracture toughness. The values represent an ensemble average over many simulations.

Poisson ratio for the solid was one quarter. Furthermore, the time step of the simulations was chosen so that $k \alpha_{\mathrm{f}} c_{\mathrm{f}} \Delta t=\pi / 10$, and the correlation function for the heterogeneity was as above, with $b=v_{0} / k \alpha_{\mathrm{f}} c_{\mathrm{f}}$.

The expected variance of the crack shape grows linearly with time, a consequence shown in Morrissey and Rice (1998) to be required due to existence of the persistent crack front waves.

\section{Summary}

This paper developed a methodology for simulating mode I fracture through regions of heterogeneous critical energy release rate. The methodology is based on the mode I perturbation analysis of Willis and Movchan (1995), as formulated by Ramanathan and Fisher (1997), and is similar in form to that of Rice et al. (1994) for a model scalar elastic solid. Simulations using the methodology developed here clearly show the effects of persistent crack front waves discussed by Morrissey and Rice (1996, 1998), including linear growth of the variance in crack shape when a crack propagates through a region of sustained, random fluctuations in critical energy release rate. However, it is evident that, in simulations for which the temporal spectral content of the toughness heterogeneity can be characterized by a small set of discrete frequencies, the effect of persistent crack front waves can be 
greatly reduced, or can be greatly enhanced for particular choices of the set of discrete frequencies.

\section{Acknowledgements}

The studies were supported by the Office of Naval Research through grant N00014-96-10777 to Harvard University, and by a Blaise Pascal Chair of the Foundation of Ecole Normale Superieure, Paris, to JRR. We thank Michael Falk for a check of our calculations which led to a revision of Figs. 2 and 3.

\section{Appendix A. Equivalence of $g^{\prime}\left(v_{0}\right) / g\left(v_{0}\right)$ and $C_{v}\left(v_{0}\right)$}

This appendix establishes the equivalence of the result for $g^{\prime}\left(v_{0}\right) / g\left(v_{0}\right)$, appearing in Eq. (1) and expressed in Eq. (17) based on Freund (1972), and the result for $C_{v}\left(v_{0}\right)$ derived in Eq. (13a) of the present work. Let $h(\xi)=(2-\xi)^{2}+4(\xi-1)^{1 / 2}(a \xi-1)^{1 / 2}$, where $a=c_{\mathrm{s}}^{2} / c_{\mathrm{d}}^{2}$. In the complex $\xi$-plane, a branch cut is taken along the real axis from 1 to $1 / a$. The function $h(\xi)$ has zeroes at the origin and on the real axis at the point $\gamma=c_{\mathrm{R}}^{2} / c_{\mathrm{s}}^{2}$.

With the branch thus defined, on the interval $[0,1]$ of the real axis $h(\xi)$ becomes, with $\xi=x$,

$$
h(x)=(2-x)^{2}-4 \sqrt{1-x} \sqrt{1-a x}=-r\left(c_{\mathrm{s}} \sqrt{x}\right),
$$

so that $r(v)=-h\left(v^{2} / c_{\mathrm{s}}^{2}\right)$. Thus,

$$
\frac{r^{\prime}(v)}{r(v)}=\left(\frac{2 v}{c_{\mathrm{s}}^{2}}\right)\left[\left.\frac{\mathrm{d} h(\xi) / \mathrm{d} \xi}{h(\xi)}\right|_{\xi=v^{2} / c_{\mathrm{s}}^{2}} .\right.
$$

Now let us consider the function

$$
H\left(\xi_{0}\right)=\oint_{\Gamma} \frac{h^{\prime}(\xi) / h(\xi)}{\xi-\xi_{0}} \mathrm{~d} \xi
$$

where $0<\xi_{0}<1$. The closed contour $\Gamma$ is taken in the clockwise direction about the branch cut of $h(\xi)$, as in Fig. A1. Note that

$$
\frac{h^{\prime}(\xi) / h(\xi)}{\xi-\xi_{0}}=\frac{\mathrm{d}}{\mathrm{d} \xi}\left\{\frac{\ln [h(\xi)]}{\xi-\xi_{0}}\right\}+\frac{\ln [h(\xi)]}{\left(\xi-\xi_{0}\right)^{2}},
$$

Since the branch point for $\ln [h(\xi)]$ is $\gamma(<1), \ln [h(\xi)] /\left(\xi-\xi_{0}\right)$ is single-valued on the contour, leaving us with

$$
H\left(\xi_{0}\right)=\oint_{\Gamma} \frac{\ln [h(\xi)]}{\left(\xi-\xi_{0}\right)^{2}} \mathrm{~d} \xi
$$




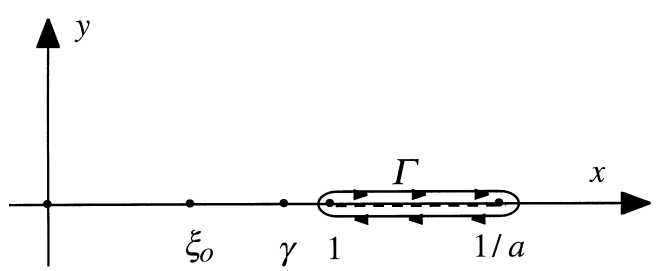

Fig. A1. Path of the contour integral in the complex $\xi$-plane defining $H\left(\xi_{0}\right)$. Note the branch cut joining the points 1 and $1 / a$ on the real axis.

Along $\Gamma_{+}$, the portion of the path $\Gamma$ lying just above the branch cut in the upperhalf of the complex $\xi$-plane, at $\xi=x$, we have

$$
\ln \left[h\left(x_{+}\right)\right]=\ln \left[\sqrt{(2-x)^{4}+16(x-1)(1-a x)}\right]+i \arctan \left[\frac{4 \sqrt{x-1} \sqrt{1-a x}}{(2-x)^{2}}\right],
$$

while along $\Gamma_{-}$, the portion of the path just below the branch cut, we have that $\ln \left[h\left(x_{-}\right)\right]$is the complex conjugate of that expression. Thus,

$$
H\left(\xi_{0}\right)=2 i \int_{1}^{1 / a} \arctan \left[\frac{4 \sqrt{x-1} \sqrt{1-a x}}{(2-x)^{2}}\right] \frac{\mathrm{d} x}{\left(x-\xi_{0}\right)^{2}} .
$$

Now $H\left(\xi_{0}\right)$, as it was originally written, will be found by application of the residue theorem. We deform the path of integration into a counter-clockwise one enclosing the roots of $h(\xi)$ as shown in Fig. A2. In the limit, as the radius of the path approaches infinity, the integral over the outer circle vanishes, leaving us with

$$
H\left(\xi_{0}\right)=2 \pi i \Sigma \text { residues at } \xi=0, \xi_{0}, \gamma .
$$

Near $\xi=0$, the integrand is

$$
\left(-\frac{1}{\xi_{0}}\right)\left(\frac{1}{1-\xi / \xi_{0}}\right) \frac{h^{\prime}(0)+h^{\prime \prime}(0) \xi+O\left(\xi^{2}\right)}{0+h^{\prime}(0) \xi+O\left(\xi^{2}\right)},
$$

giving a residue of $-1 / \xi_{0}$. Near $\xi=\xi_{0}$, the integrand is expanded as

$$
\left(\frac{1}{\xi-\xi_{0}}\right)\left[h^{\prime}\left(\xi_{0}\right) / h\left(\xi_{0}\right)+O\left(\xi-\xi_{0}\right)\right]
$$

giving a residue of $h^{\prime}\left(\xi_{0}\right) / h\left(\xi_{0}\right)$. Lastly, near $\xi=\gamma$ the integrand is expanded as

$$
\left(\frac{1}{\gamma-\xi_{0}}\right)\left[\frac{1}{1-(\xi-\gamma) /\left(\gamma-\xi_{0}\right)}\right] \frac{h^{\prime}(\gamma)+h^{\prime \prime}(\gamma)(\xi-\gamma)+O\left[(\xi-\gamma)^{2}\right]}{0+h^{\prime}(\gamma)(\xi-\gamma)+O\left[(\xi-\gamma)^{2}\right]}
$$




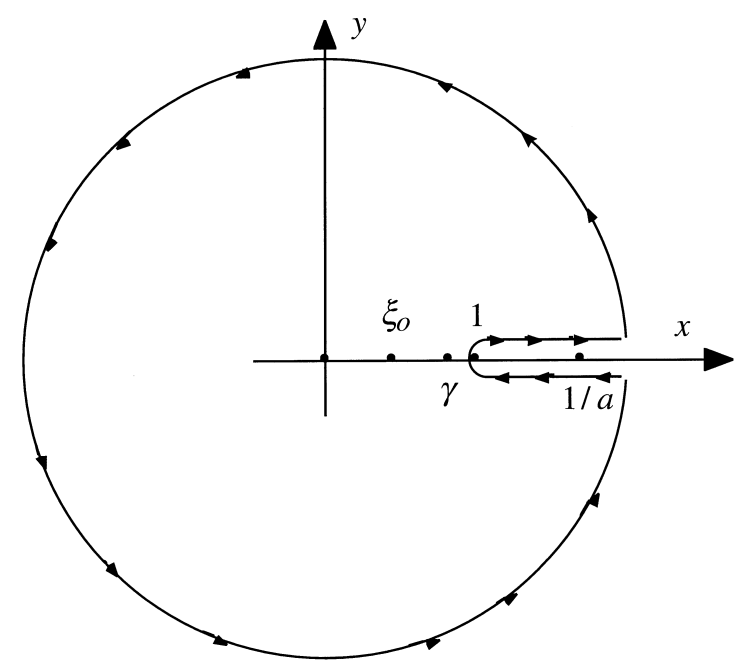

Fig. A2. Alternative path allowing evaluation of $H\left(\xi_{0}\right)$ by the residue theorem.

which leaves a residue of $1 /\left(\gamma-\xi_{0}\right)$. Thus,

$$
H\left(\xi_{0}\right)=2 \pi i\left(\frac{h^{\prime}\left(\xi_{0}\right)}{h\left(\xi_{0}\right)}+\frac{1}{\gamma-\xi_{0}}-\frac{1}{\xi_{0}}\right) .
$$

Recalling that we have developed above an integral representation of $H\left(\xi_{0}\right)$, and equating the two, we find

$$
\frac{h^{\prime}\left(\xi_{0}\right)}{h\left(\xi_{0}\right)}=\frac{1}{\xi_{0}}-\frac{1}{\gamma-\xi_{0}}+\frac{1}{\pi} \int_{1}^{1 / a} \arctan \left[\frac{4 \sqrt{x-1} \sqrt{1-a x}}{(2-x)^{2}}\right] \frac{\mathrm{d} x}{\left(x-\xi_{0}\right)^{2}} .
$$

Therefore, substituting into the expression above for $r^{\prime}(v) / r(v)$, with $v=v_{0}$, and making the change of variable $x=\left(\eta / c_{\mathrm{s}}\right)^{2}$ we are finally left with

$$
\begin{aligned}
\frac{r^{\prime}\left(v_{0}\right)}{r\left(v_{0}\right)} & =2 \frac{v_{0}}{c_{\mathrm{s}}^{2}}\left\{\frac{c_{\mathrm{s}}^{2}}{v_{0}^{2}}-\frac{1}{c_{\mathrm{R}}^{2} / c_{\mathrm{s}}^{2}-v_{0}^{2} / c_{\mathrm{s}}^{2}}\right\}+\int_{c_{\mathrm{s}}}^{c_{\mathrm{d}}} \Theta\left(\eta ; c_{\mathrm{s}}, c_{\mathrm{d}}\right) \frac{2 v_{0} \eta}{\left(\eta^{2}-v_{0}^{2}\right)^{2}} \mathrm{~d} \eta \\
& =\frac{2}{v_{0}}-\frac{2 v_{0}}{c_{\mathrm{R}}^{2}-v_{0}^{2}}+\int_{c_{\mathrm{s}}}^{c_{\mathrm{d}}} \Theta\left(\eta ; c_{\mathrm{s}}, c_{\mathrm{d}}\right) \frac{2 v_{0} \eta}{\left(\eta^{2}-v_{0}^{2}\right)^{2}} \mathrm{~d} \eta
\end{aligned}
$$

Combining this with the other terms of $g^{\prime}\left(v_{0}\right) / g\left(v_{0}\right)$ in Eq. (17) then gives

$$
\frac{g^{\prime}\left(v_{0}\right)}{g\left(v_{0}\right)}=\frac{c_{\mathrm{d}}}{c_{\mathrm{d}}^{2}-v_{0}^{2}}-\frac{2 c_{\mathrm{R}}}{c_{\mathrm{R}}^{2}-v_{0}^{2}}+\int_{c_{\mathrm{s}}}^{c_{\mathrm{d}}} \Theta\left(\eta ; c_{\mathrm{s}}, c_{\mathrm{d}}\right) \frac{\eta^{2}+v_{0}^{2}}{\left(\eta^{2}-v_{0}^{2}\right)^{2}} \mathrm{~d} \eta,
$$

which coincides with our expression for $C_{v}\left(v_{0}\right)$ in Eq. (13a) and proves the result. 


\section{References}

Freund, L.B., 1972. Crack propagation in an elastic solid subject to general loading, I, constant rate of extension, II, non-Uniform rate of extension. J. Mech. Phys. Solids 20, 129-152.

Geubelle, P.H., Rice, J.R., 1995. A spectral method for three-dimensional elastodynamic fracture problems. J. Mech. Phys. Solids 43, 1791-1824.

Ramanathan, S., Fisher, D.S., 1997. Dynamics and instabilities of planar tensile cracks in heterogeneous media. Phys. Rev. Lett. 79, 877.

Morrissey, J.W., Rice, J.R., 1996. 3D elastodynamics of cracking through heterogeneous solids: crack front waves and growth of fluctuations (abstract). EOS, Trans. Amer. Geophys. Union 77 (46, Fall Mtg. Suppl.), F485.

Morrissey, J.W., Rice, J.R., 1998. Crack front waves. J. Mech. Phys. Solids 46, 467-487.

Perrin, G., Rice, J.R., 1994. Disordering of a dynamic planar crack front in a model elastic medium of randomly variable toughness. J. Mech. Phys. Solids 42, 1047-1064.

Rice, J.R., Ben-Zion, Y., Kim, K.S., 1994. Three-dimensional perturbation solution for a dynamic planar crack moving unsteadily in a model elastic solid. J. Mech. Phys. Solids 42, 813.

Willis, J.R., Movchan, A.B., 1995. Dynamic weight functions for a moving crack I. Mode I loading. J. Mech. Phys. Solids 43, 319. 